\title{
The Doppler Spectra of Medium Grazing Angle Sea Clutter; Part 1: Characterisation
}

Simon Watts ${ }^{1,4}$, Luke Rosenberg ${ }^{2,3}$, Stephen Bocquet ${ }^{2}$, Matthew Ritchie ${ }^{4}$

${ }^{1}$ Consultant, United Kingdom

${ }^{2}$ Defence Science and Technology Organisation, Australia

${ }^{3}$ University of Adelaide, Australia

${ }^{4}$ UCL, London, United Kingdom

Email: simon.watts.gb@ieee.org

Abstract - This paper is concerned with the characterisation of Doppler spectra from high range resolution X-band radar sea clutter observed from an airborne platform over the range of grazing angles, $15^{\circ}$ to $45^{\circ}$. It is observed that when looking up or down wind there is a strong correlation between mean Doppler shift and local spectrum intensity. When combined with random fluctuations of spectrum width, these characteristics give the spectra a temporal and spatial variability. This behaviour has previously been observed in low grazing angle data and these results confirm the wider applicability of the models developed using that data. The modelling method is also extended here to capture the bimodal behaviour observed with high intensity returns from breaking waves looking up or down-wind.

Keywords-Radar sea clutter, Doppler spectra, modelling and simulation

\section{Introduction}

Models of the returns from radar sea clutter are an important tool in the development of maritime radars [1]. Models of average reflectivity, characterized by the normalized radar cross-section, $\sigma^{0}$, have been widely reported over many years [2-5]. Models of amplitude statistics are now mainly based on various compound formulations of the probability density function (PDF) of the clutter amplitude or intensity, such as the compound K distribution [5] and more recently the Pareto [6][7][8] and K-plus-Rayleigh models [9]. The modelling of Doppler spectra has been less well developed. Empirical models of mean Doppler shift and spectrum width as a function of wind speed were developed by [10] and summarised in [3] along with other measurements. However, suitable models that capture the time and/or spatially varying nature of the Doppler spectra, and that can be used to simulate realistic coherent clutter returns and to predict the performance radar detection algorithms, were not developed until quite recently. Some work on the simulation of realistic Doppler spectra had been previously reported in [11] and other potential methods have also previously been proposed, such as [12]. Those methods can go some way towards reproducing examples of Doppler spectra but do not allow the generation of continuous coherent returns from which realistic spectra can be derived. 
Numerical studies based on electro-magnetic scattering from a simulated sea surface are starting to contribute to the understanding of the characteristics of range-resolved Doppler spectra of sea clutter [13]. This type of modelling still cannot reproduce all the characteristics of Doppler spectra that are of interest. However, it does start to explain some of the mechanisms that may contribute to the variations in mean Doppler shift and spectrum width that are discussed in this paper.

Based on wave-tank measurements, Walker [14][15]developed a method of modelling the average Doppler spectrum, based on Gaussian-shaped components of the power spectral density (PSD). These components, with different mean Doppler frequency, spectrum width and intensity, were associated with three different scattering mechanisms (Bragg, whitecap and burst scattering). However, this approach does not model the short-term temporal variations of the Doppler spectra, which give rise to non-Gaussian amplitude statistics in the spectrum edges. This behaviour was reported originally in $[16]$, and more recently in $[5][17]$, and it is this characteristic that is particularly problematic when trying to set detection thresholds to give a constant false alarm rate. This apparently non-stationary behaviour [18][19]also means that characterizing the Doppler spectrum by a covariance matrix of the speckle component that is derived by averaging over a long time period may not correctly represent its temporal variations.

A model that can represent the time-varying nature of Doppler spectra in sea clutter was originally described in [20] and then further expanded in [21] For sea clutter with grazing angles $<2^{\circ}$, it was observed that the mean Doppler shift of the power spectrum was strongly correlated with the local intensity of the clutter return. When the local intensity is modelled with a suitable temporal or spatial correlation and a probability density function, such as the compound $\mathrm{K}$ distribution, with added thermal noise, it is possible to predict the typical amplitude statistics in individual Doppler bins, as observed over a period of time or over a range interval. This model can be used to simulate time-varying Doppler spectra and to analytically investigate the performance of signal processing techniques [22] The work in [21] has been extended with further analysis of the same data set to show how the Doppler spectra characteristics vary with wind or wave direction [23]

It is of considerable interest to test this new modelling method on a wider range of data. In this paper, both dual and fully polarised sea clutter data gathered by the DSTO Ingara radar are analysed. An important feature of this data is that it is gathered at higher grazing angles than $[21]$, covering from $15^{\circ}$ to $45^{\circ}$. The data also covers all combinations of horizontal and vertical polarisations where $\mathrm{H}$ and $\mathrm{V}$ represent the horizontal and vertical channels respectively. Initial results of this work were reported in $[24]$ and this paper provides further details of the results achieved. Section 2 describes the Ingara data and outlines how it was analysed to extract the characteristics required for the modelling. Section 3 provides details of the modelling methods used to characterise the spectra, including the 
methods used to capture the bimodal behaviour observed in some conditions. The purpose of this modelling is to improve the analytic prediction of radar performance and to allow coherent radar data to be realistically simulated. Therefore, these models are only of value if they can accurately capture the salient properties of real clutter spectra. These aspects of this work are further explored in Part 2 of this paper [25]

\section{Data Analysis}

\subsection{Ingara Data}

Ingara is a fully-polarimetric X-band radar system maintained and operated within the DSTO in Australia [26], The dataset comprises two trials on separate occasions and in two distinctly different regions. The first sea-clutter trial was conducted in 2004 in the Southern Ocean approximately $100 \mathrm{~km}$ south of Port Lincoln, South Australia. The site chosen was at the edge of the South Australian continental shelf where there was little chance of shallow water affecting the wave field. During the trial, ocean backscatter was collected for a range of different geometries on eight separate days with different ocean conditions. The second trial in 2006 was conducted in littoral and open ocean environments near Darwin in the Northern Territory.

During the ocean backscatter collections, Ingara was operated in the circular spotlight-mode (see [26]). In this mode, the aircraft flies a circular orbit in an anti-clockwise direction (as seen from above) around a nominated point of interest, while the radar beam is continuously directed toward this point. Each day the radar platform performed at least six full orbits around the same patch of ocean to cover a large portion of grazing angles between $15^{\circ}$ and $45^{\circ}$. The Ingara data analysed has a $200 \mathrm{MHz}$ bandwidth $(0.75 \mathrm{~m}$ range resolution) centred at $10.1 \mathrm{GHz}$. For the dual polarised (dual-pol) mode, the radar transmits with either a horizontal or vertical polarisation and then receives both polarisations. The fully polarised (full-pol) mode alternated transmitting horizontal and vertical polarisations while receiving both simultaneously. This mode of operation halved the effective pulse repetition frequency (PRF).

Before the data was analysed, a number of pre-processing steps were applied. Firstly, the sampled signal was processed to remove bandpass filter modulations and adjusted for motion compensation using both the inertial navigation unit and the global positioning system on board the radar platform. The next steps included a correction for the variation in ground range resolution due to changes in grazing angle, removal of the elevation beam pattern and polarimetric calibration using the procedure described in [27],

The mean instantaneous thermal noise power of the radar system, $p_{n}$, was found by processing part of the collection where the RF transmitter was turned off. By assuming the sea-clutter and thermal noise are independent throughout the processing chain, a noise signal was then created in the backscatter coefficient domain with the same pre- 
processing steps applied as the clutter plus noise signal. This ensures there is an accurate measure of the noise component of the radar backscatter (clutter plus noise).

To demonstrate the characterisation and modelling, dual-pol data from the 2006 trial has been processed over 9 bursts of 64 pulses covering an aspect angle range of about $1.8^{\circ}$. The data spans $29^{\circ}-38^{\circ}$ grazing and was analysed over groups of 200 range bins, each representing a range swath of $150 \mathrm{~m}$ with a variation of grazing angles of less than $2^{\circ}$ over the swath. In all cases the power spectra at each range gate and for each burst were estimated using a 64 point FFT with a $-55 \mathrm{~dB}$ Dolph-Chebyshev weighting, as in [21]. An example of the data, presented as a range-time magnitude plot over $750 \mathrm{~m}$ (1017 range cells) and 576 pulses, for HH polarised data, looking up-wind, is shown in Fig. 1(i). A second figure showing the Doppler spectra for the first 64 pulses is then shown in Fig. 1(ii). The range varying nature of the spectra is very evident from this illustration.

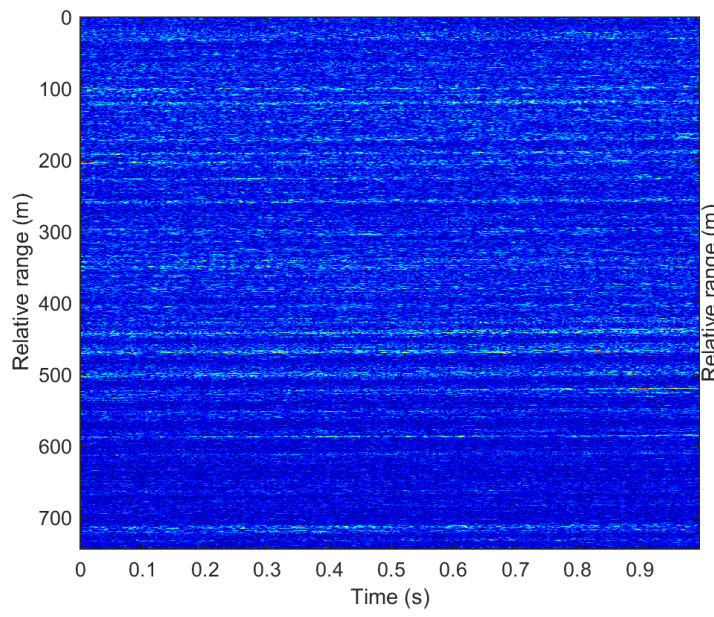

(i)

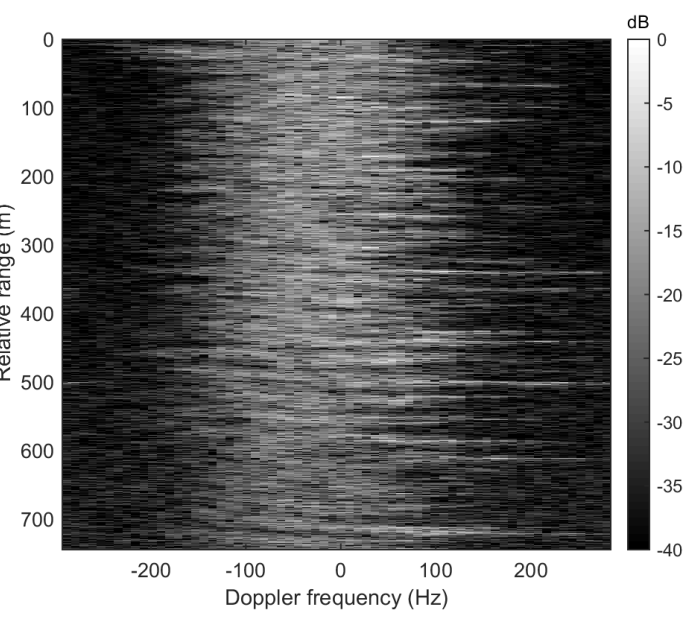

(ii)

Figure 1 (i) : Range-time magnitude image for the $\mathrm{HH}$ polarisation; (ii) Range-Doppler image for the $\mathrm{HH}$ polarisation

This Ingara data was analysed to assess whether the basic model for Doppler spectra proposed in $[21]$ would still be applicable to data from higher grazing angles. The key features of the data are given in Table 1 and the essential parameters required to define the models are:

- $\quad$ PDF of the pulse returns 
- The shape of the Doppler power spectra

- The clutter-to-noise ratio (CNR) of the pulse returns.

- The mean Doppler shift of the Doppler spectra and any correlation with local intensity

- The width of the normalised spectra (measured as the standard deviation of the power spectrum)

Table 1 Ingara dual-pol data characteristics

\begin{tabular}{|l|l|}
\hline Polarisations & $\mathrm{HH}, \mathrm{HV}, \mathrm{VH}$ and VV \\
\hline Range resolution & $0.75 \mathrm{~m}$ \\
\hline Range coverage & 3.0 to $3.65 \mathrm{~km}$ (typical) \\
\hline Grazing angles & $29^{\circ}$ to $38^{\circ}$ \\
\hline Azimuth/Elevation beamwidth & $1^{\circ} / 13^{\circ}$ (two-way, $\left.3 \mathrm{~dB}\right)$ \\
\hline Frequency & $10.1 \mathrm{GHz}$ \\
\hline PRF & $578 \mathrm{~Hz}$ \\
\hline Aircraft speed & $100 \mathrm{~m} / \mathrm{s}(200 \mathrm{kts})$ \\
\hline
\end{tabular}

\subsection{PDF of Pulse Returns}

The analysis of the PDF of the data has shown the interesting result that even at these medium grazing angles the $\mathrm{HH}$ polarized data can appear very spiky in nature. The periodic variation of reflectivity with range due to the sea wave pattern is evident in Fig. 1(i). A small section of this data is displayed in Fig. 2. It can be seen here that for a duration of about 15 pulses in range bin 279 , a very large spike has appeared. This spike has a peak intensity that is about 16 times greater than the mean of the surrounding clutter. It is also very short-lived at this intensity, lasting about 16 pulses ( $\sim 25 \mathrm{~ms})$. However, it can also be seen that it is associated with an area of the sea surface where the intensity is generally higher than in adjacent range cells.

A single large spike such as this can have a significant effect on attempts to fit data to a PDF model. Fig. 3 compares two attempts to fit to a set of data taken from range bins 201 to 400 (see Fig. 1(ii)) over two successive bursts of 64 pulses. The first burst contains the larger spike. It can be seen that attempts to fit to a $\mathrm{K}+$ noise model (see, for example [5]) fail because of the presence of the spike. However, on the second burst, occurring only about $10 \mathrm{~ms}$ later in time, the fit is quite good. When averaged over a larger data sample the effects of these rare spikes in the $\mathrm{HH}$ data are reduced but it was found that a $\mathrm{K}+$ noise model did not fit the $\mathrm{HH}$ data very well. Whilst the compound $\mathrm{K}$ distribution model fits the VV Ingara data quite well [28], a better fit to the HH and HV/VH data may often be obtained with a Pareto-plus-noise distribution or a K-plus-Rayleigh distribution [9].

For the purposes of illustration here the $\mathrm{K}$ distribution PDF, with added thermal noise, will be used but the results can readily be extended to incorporate alternative PDF models (see for example, [29]). 


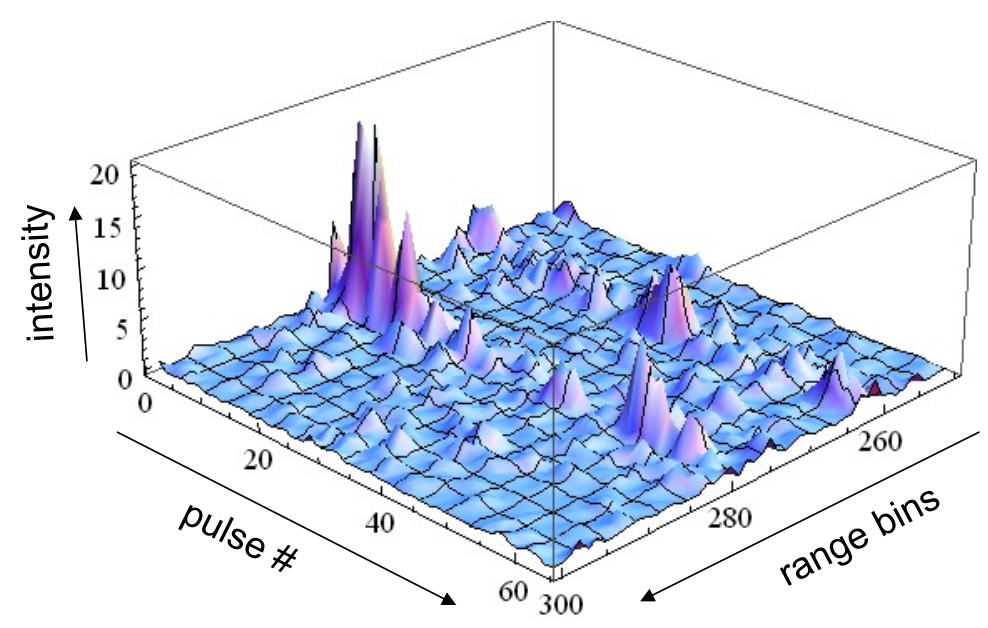

Figure 2 Intensity of returns in range bins 250 to 300 for a burst of 64 pulses

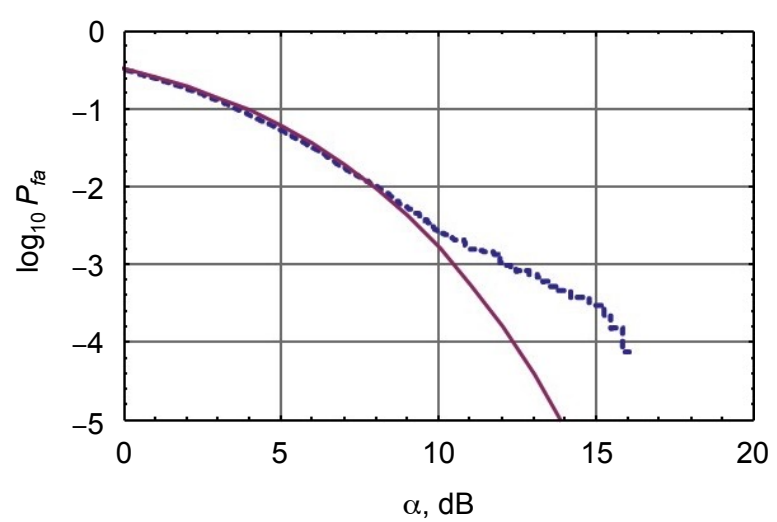

(a)

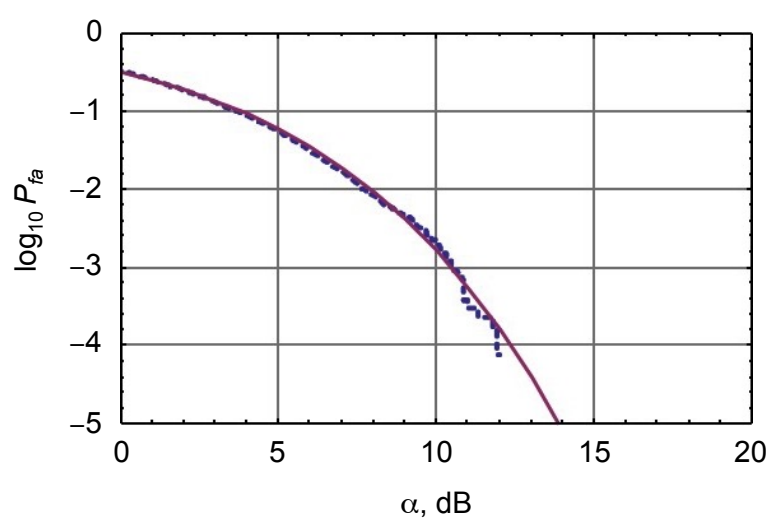

(b)

Figure 3 Cumulative distributions of data from 200 range cells over 2 successive bursts of 64 pulses; a : burst with large clutter spike; b: burst with no large spikes.

\subsection{Shape of Doppler Spectra}

The analysis in [21] found that individual Doppler spectra could be well represented by Gaussian-shaped power spectral densities, with added thermal noise. The Ingara data analysed here showed similar characteristics at low and intermediate intensity levels, with some deviation from a simple Gaussian shape at high intensity levels.

To investigate the spectrum shapes, normalised power spectra were estimated. The normalisation consisted of shifting the mean Doppler to $0 \mathrm{~Hz}$ and normalising the spectrum intensity to a peak value of unity. The normalised spectrum for the upwind HH data, averaged over 200 range bins, is shown in Fig. 4(i). Also shown is a Gaussian shape having the same average spectrum width (standard deviation). It can be seen that the Gaussian shape is a good approximation to the average shape, with some deviation at the edges of the spectrum at higher values of Doppler shift. This deviation is due to the bimodal behaviour. 
The spectra observed here have been broadened by the aircraft motion, as discussed in $[24]$ The underlying seaclutter Doppler spectra are convolved with a Doppler spread determined by the antenna beam pattern, the look direction and the platform speed.

It was observed that spectra with very high intensities relative to the overall mean had a broader shape. This is illustrated in Fig. 4(ii), which shows the average spectra (not shifted in frequency) for selected range samples where the power in the Doppler bin centred on $168.75 \mathrm{~Hz}$ (chosen empirically; see Fig. 4(ii)) exceed 4 times the mean power in that bin over the range interval. In this example, about $5 \%$ of the power spectra met this magnitude criterion. It can be seen that this data does not have a simple Gaussian shape, but appears to exhibit a bimodal structure. Also shown is a Gaussian shape having the same mean Doppler shift and mean standard deviation as the data, which is not a good fit due to the bimodal nature of the spectra. By contrast, Fig. 4(iii) shows the averaged spectra for the remaining $95 \%$ of the range samples in that burst, which clearly has a more regular Gaussian shape.

The bimodal behaviour in this data has been reported by Rosenberg [30], who analysed the fit of the mean Doppler spectra with a two component Gaussian model. This apparent bimodal behaviour can be observed in the data in areas of high clutter intensity, as illustrated in Fig. 2. The power spectrum of the data in Fig. 2 is shown in Fig. 5. In Fig. 2 it can be seen that there are regions of higher intensity clutter in range bins 262 and 279 . The power spectra in these regions have a much higher mean Doppler shift than the surrounding range bins and appear much broader. It can also be observed that a very large peak in the Doppler domain has occurred at a frequency of about $150 \mathrm{~Hz}$. The very large spike in the time domain at range 279 has not resulted in such a large peak in the Doppler domain, although clearly the power in the spectrum at this range is much higher than the surrounding range cells and the spectrum is much broader. The intensity-dependent nature of this apparent bimodal behaviour needs to be captured in any models of behaviour. A particular method that has been developed here is described below. 


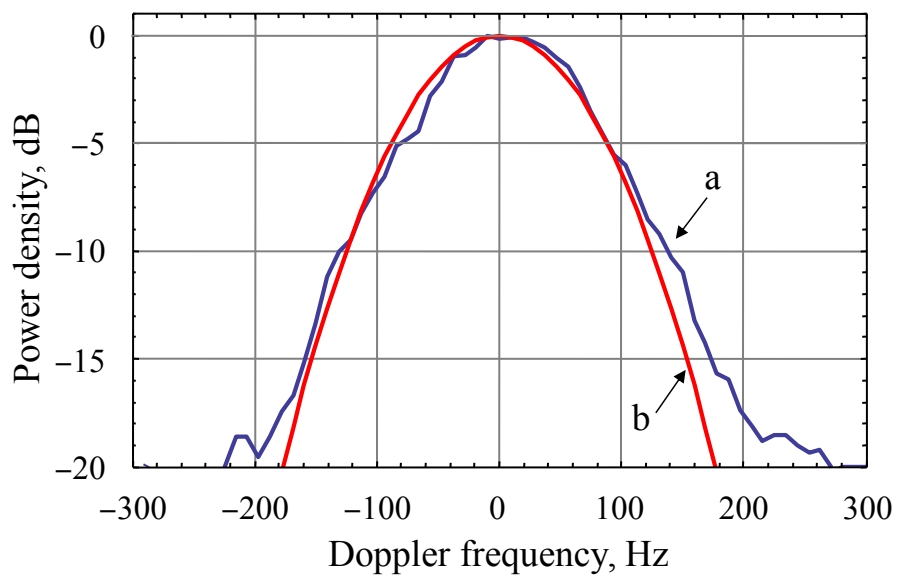

(i)

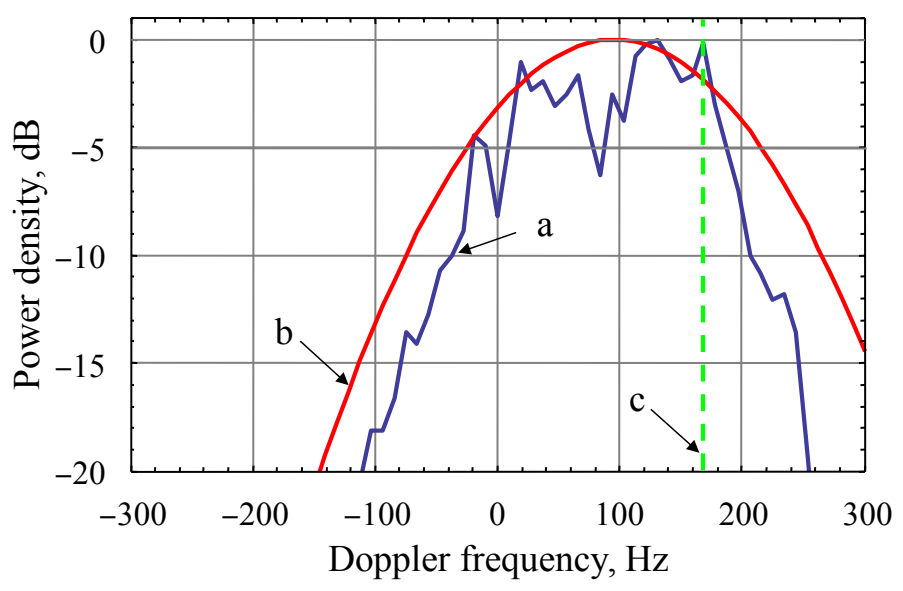

(ii)

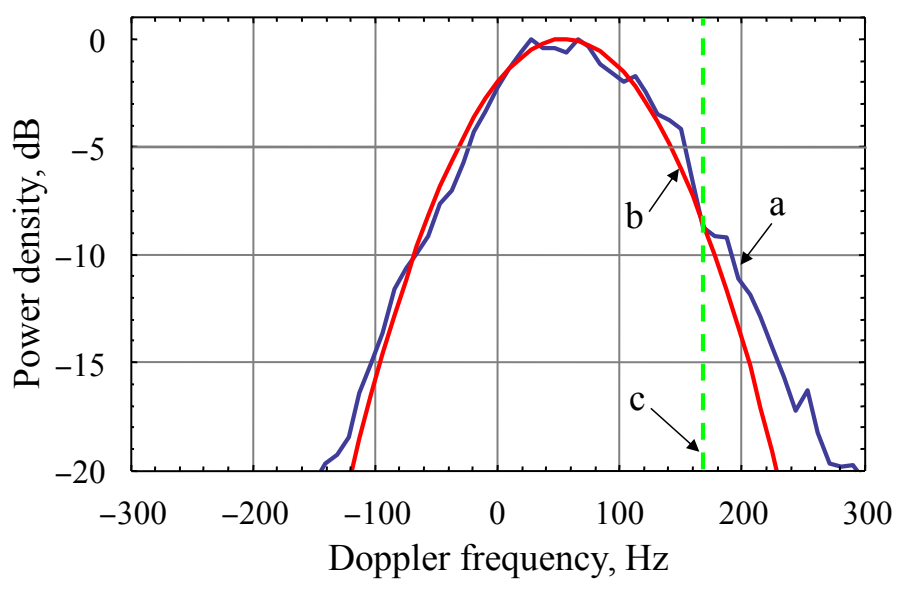

(iii)

Figure 4 (i): Average normalised spectrum shape. a: $\mathrm{HH}$ pol, upwind data; b: mean Gaussian shape fitted to data.; (ii) Average power spectra for high values of intensity. a: HH pol, upwind data; b: mean Gaussian shape fitted to data; c: Doppler bin centred on $168.75 \mathrm{~Hz}$ used to select bimodal spectra.; (iii) Average spectra for lower values of intensity. a: HH pol, upwind data; b: mean Gaussian shape fitted to data; c: Doppler bin centred on $168.75 \mathrm{~Hz}$ used to select bimodal spectra. 


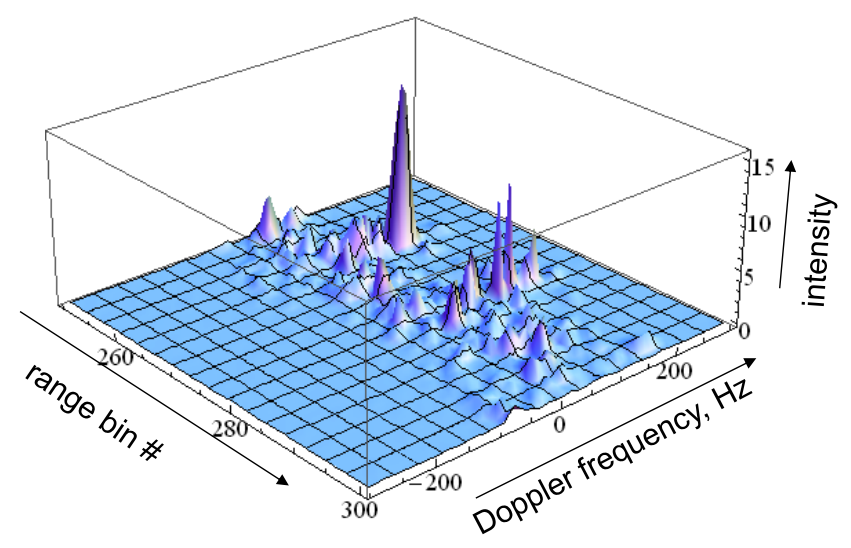

Figure 5 Doppler spectra of returns in range bins 250 to 300 for a burst of 64 pulses

The data here has been analysed using 64 point FFTs. It is also of interest to consider other FFT lengths, which may give some insight into the Doppler characteristics of the large time-domain spikes. Fig. 6(i) shows the evolution of the spectrum in range bin 279, using successive 16 point FFTs, overlapped by 12 samples over a total time period of 200 pulses. A burst of 16 pulses is approximately the lifetime of the large spike shown in Fig. 2. It can be seen that the large spike has a centre frequency that is close to $150 \mathrm{~Hz}$ and that the clutter at this range has a high mean Doppler that persists over time. In the neighbouring range bin 282, shown in Fig. 6(ii), the clutter has a much lower intensity and a lower mean Doppler.

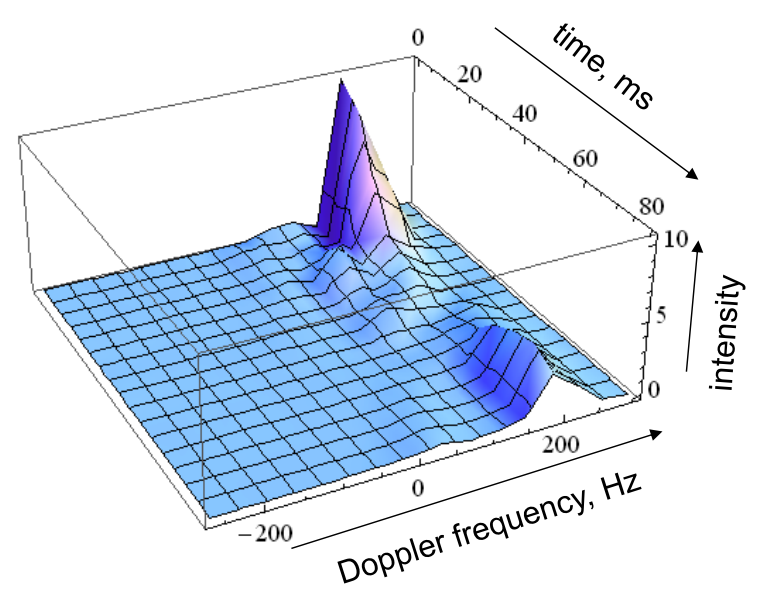

(i)

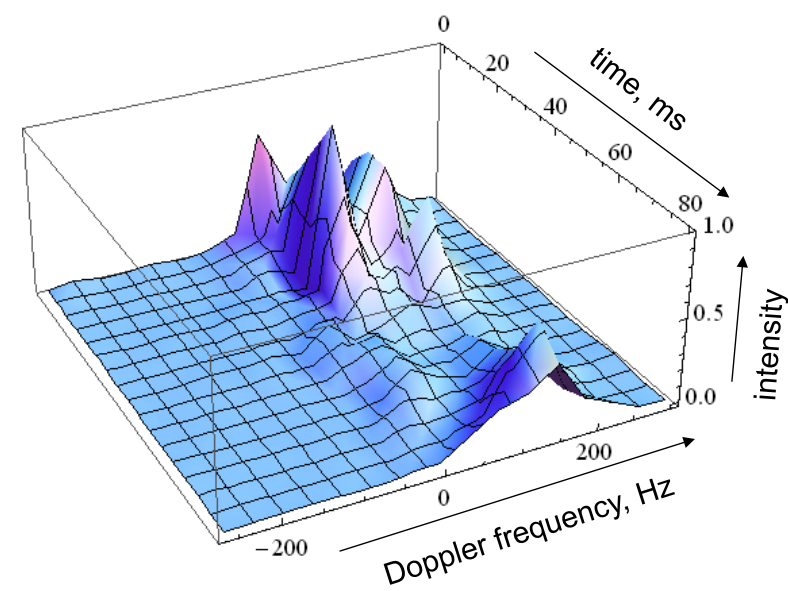

(ii)

Figure 6 (i):Spectrum of spike in range bin 279, measured with a 16 point FFT, as it evolves over time; (ii) Spectrum of lower intensity clutter in range bin 282 , measured with a 16 point FFT, as it evolves over time. 


\subsection{Mean Doppler shift and spectrum width}

The application of any new model requires certain characteristics of the spectra to be measured. The mean Doppler shift and normalised width (standard deviation of the power spectrum) were estimated for the spectra in each range bin over a range interval. In order to estimate the parameters of the spectra in this way, it is desirable to have a high clutter-to-noise ratio (CNR). For the analysis here, the data generally had a CNR of at least $3 \mathrm{~dB}$ and a simple correction was applied to the estimates of the mean frequency, $m_{f}(k)$, and variance, $\widetilde{\sigma}_{f}^{2}(k)$, of the power spectrum in the $k$ th range cell, as shown below. The spectra where obtained by using an FFT of length N, corresponding to the pulse burst length (mainly $\mathrm{N}=64$ here). The moments were estimated using

$$
\begin{aligned}
& m_{f}(k)=\frac{1}{h} \sum_{n=1}^{N}\left(\frac{n f_{r}}{N}-\frac{f_{r}}{2}\right) S(n, k) \\
& \widetilde{\sigma}_{f}^{2}(k)=\frac{1}{h}\left(\sum_{n=1}^{N}\left(\frac{n f_{r}}{N}-\frac{f_{r}}{2}-m_{f}(k)\right)^{2} S(n, k)-\frac{S_{\text {noise }} f_{r}^{3}}{12}\right)
\end{aligned}
$$

where $h=\left(\sum_{n=1}^{N} S(n, k)-S_{\text {noise }} f_{r}\right), n$ is the FFT bin number, $S_{\text {noise }}$ is the power spectral density of the noise, $f_{r}$ is the PRF, and $S(n, k)$ is the power spectral density of clutter-plus-noise for the $n$th Doppler bin and $k$ th range cell. This method is satisfactory provided that the CNR is not too low. If the CNR is $<3 \mathrm{~dB}$, it is usually better not to correct the estimates of the moments, but to recall that clutter-plus-noise is being measured. As CNR $\rightarrow 0$, the value of $h \rightarrow 0$ and the moments as defined in (1) tend to $\infty$. If the noise is not corrected, then as CNR $\rightarrow 0, m_{f}(k) \rightarrow 0$ and $\widetilde{\sigma}_{f}^{2}(k) \rightarrow f_{r}^{2} / 12$.

The parameter $\widetilde{\sigma}_{f}^{2}(k)$ estimated from the data includes the effects of platform motion. To account for the spreading due to platform motion, a Gaussian shape was fitted to the Ingara azimuth beam pattern. For a side-looking antenna and platform velocity, $v$, the effect of platform motion can then be modelled as imparting a Gaussian-shaped spectrum on any point reflector as the antenna scans past it. If $\phi_{3 \mathrm{~dB}}$ is the two-way $3 \mathrm{~dB}$ azimuth beamwidth, then the spectrum component due to platform motion has a zero mean and variance of

$$
\sigma_{\text {antenna }}^{2}=\frac{v \phi_{3 d B} \cos \theta}{\lambda \sqrt{2 \log _{e} 2}}
$$

where $\theta$ is the elevation angle and is equivalent to the grazing angle for a flat earth with no refraction due to atmospheric effects. For clutter returns, the inherent clutter spectrum is modified by being convolved with the spectrum imparted by the platform motion. The underlying clutter spectrum can then be modelled as a Gaussian with variance given by 


$$
\sigma_{f}^{2}(k)=\widetilde{\sigma}_{f}^{2}(k)-\sigma_{\text {antenna }}^{2}
$$

To see the distribution of the underlying spectral widths and centre points, the formulation in (1) has been applied to each range bin, $k$ which can be associated with the normalised local clutter intensity, $x_{n}=\mathrm{x} /<\mathrm{x}>$, approximately given by the total power of the spectrum. We can now plot $m_{f}\left(x_{n}\right)$ as a function of $x_{n}$ to investigate any correlation. Fig. 7 shows this for data taken from 200 range cells over 9 bursts of 64 pulses, for the HH, HV and VV polarisations. Fig. 8 shows the equivalent plot for the standard deviation $\sigma_{f}\left(x_{n}\right)$.

It can be seen from Fig. 7 that there appears to be a strong correlation between the mean Doppler shift and the spectrum intensity, although this variation is quite noisy. The correlation would appear, at least qualitatively, to be linear for low and high values of intensity, but with different slopes in these two regions. Conversely, in Fig. 8 there does not appear to be a strong correlation between the spectrum standard deviation and the intensity, although there is some suggestion of a decrease in spectrum width at higher levels of intensity. The model predictions for this data, also shown in the Figures, are explained in Section 3.1. As reported in $[21]$ it is expected that the dependence of $m_{f}\left(x_{n}\right)$ on intensity, $x_{n}$, will depend on the wind or wave direction. The dependence on wind direction is discussed further in Section 3.2 .

It is interesting to note that variations in mean Doppler shift over a range and fluctuations in spectrum width have been predicted by numerical studies of electro-magnetic backscatter from simulated sea surfaces [13]. The variation in mean Doppler shift is associated with the variation in "long-wave" orbital velocity of the surface projected along the radar line of sight. The width of the Doppler spectra is associated with the variation over the observation time of the projected surface orbital velocities at a given range.
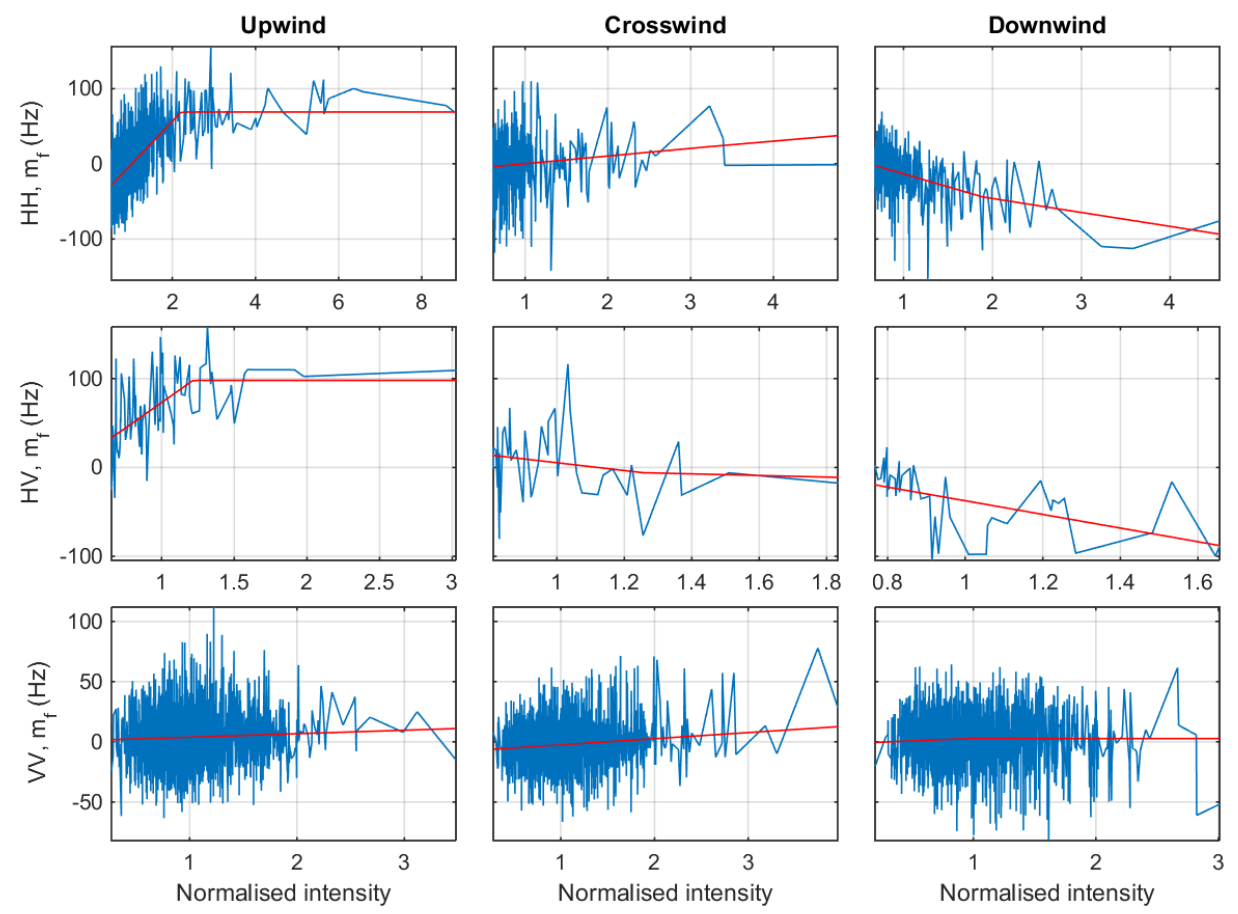
Figure 7 Mean Doppler shift vs. normalised spectrum intensity: blue - data, red - model prediction (section 3.1).
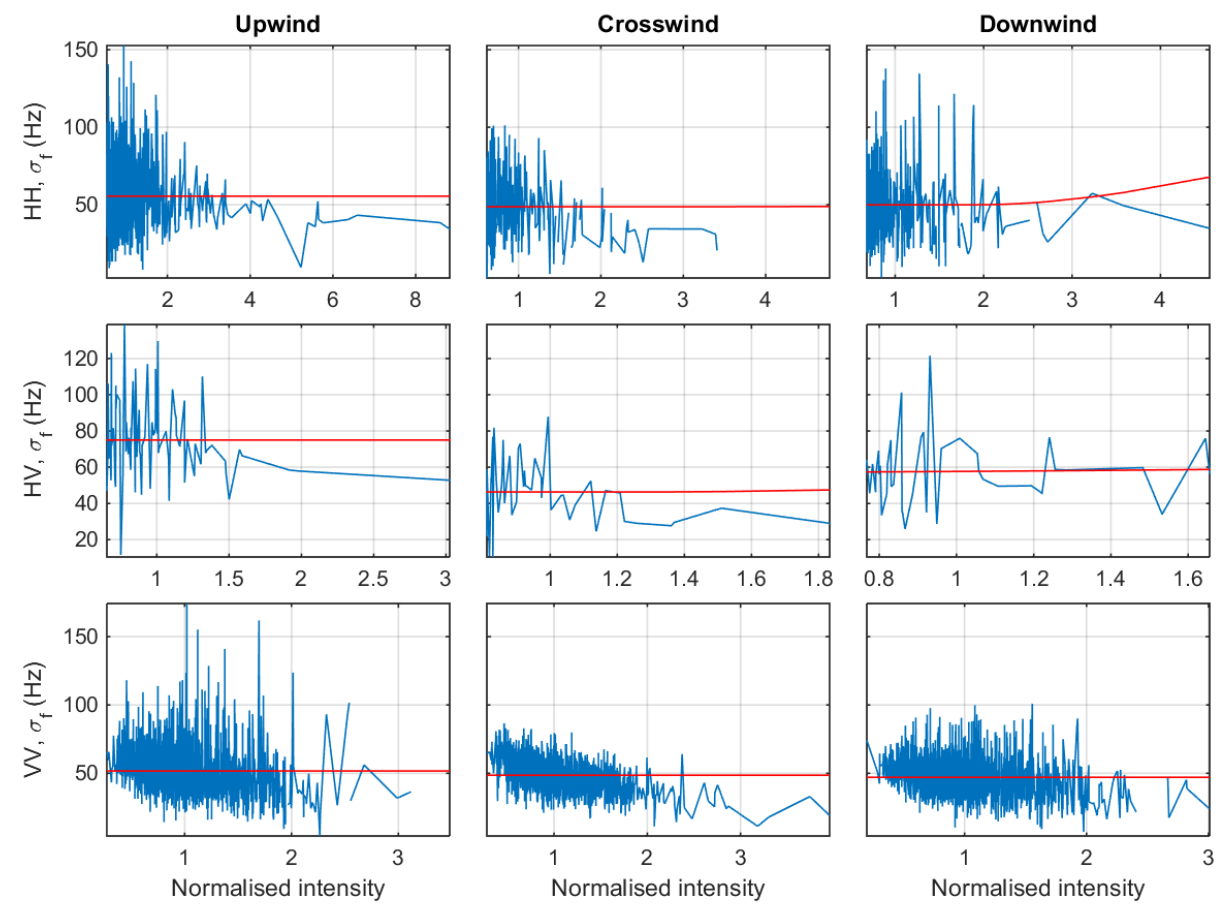

Figure 8 Spectrum standard deviation vs. intensity: blue - data, red - model prediction (section 3.1).

\section{Modelling of spectra}

\subsection{Bimodal Spectra}

Rosenberg [30] showed that a good fit to the power spectrum averaged over a range interval could be obtained by a mixture of two Gaussians. It was assumed that these two components could be attributed to Bragg scattering and fast components of the clutter return, similar to the mechanisms suggested by Walker [14][15], In the analysis presented in this paper, we are interested in the short-term variation of the spectra, as the local mean intensity varies. As discussed above, the bimodal behaviour reported in [30] appears to be associated with high levels of local intensity relative to the overall mean level (see Fig. 7). This idea is incorporated into the modelling described below. The original model for $m_{f}\left(x_{n}\right)[21]$ proposed a fit of the form

$$
m_{f}\left(x_{n}\right)=A+B x_{n}
$$

It can be seen from the examples in Fig. 7 that this may still be a valid model. As noted in $[21]$ the variation of $m_{f}\left(x_{n}\right)$ with $x_{n}$ is quite noisy in the real data, but the simple relationship in (4) appears to capture the salient features of the spectra in subsequent modelling. The argument that despite its noisy appearance this linear relationship is valid is further strengthened by the clear trends seen in how this linear fit varies with azimuth angle in [31], These trends show that fundamentally the linear fit is capturing a real relationship between the PSD centre and the clutter intensity. The spread of values about the mean $m_{f}\left(x_{n}\right)$ associated with a given value of $x$ has also been modelled, as discussed above. It is found that a Gaussian probability density function is a reasonable fit to this parameter. The clutter mean Doppler can now be modelled with the form 


$$
m_{f}\left(x_{n}\right)=A+B x_{n}+r
$$

where $r$ is a Gaussian random variable with zero mean and standard deviation, $\sigma_{r}$, having a pdf $p_{r}(r)$. It is also observed here that in some cases for high values of normalised intensity the rate of increase of $m_{f}\left(x_{n}\right)$ with $x_{n}$ appears to reduce, when looking up or down-wind. It is proposed here that this observation can be explained by increasingly bimodal behaviour above some level of intensity. A possible general model for the power spectrum of the clutter alone (i.e. without the added thermal noise) is given by a mixture model of two Gaussian-shaped power spectra:

$$
\begin{aligned}
\mathbf{G}\left(f, x_{n}, s_{1}, s_{2}\right)= & \frac{\alpha x_{n}}{\sqrt{2 \pi} s_{1}} \exp \left[-\frac{\left(f-m_{f 1}\left(x_{n}\right)\right)^{2}}{2 s_{1}^{2}}\right] \\
& +\frac{\beta x_{n}}{\sqrt{2 \pi} s_{2}} \exp \left[-\frac{\left(f-m_{f 2}\left(x_{n}\right)\right)^{2}}{2 s_{2}^{2}}\right]
\end{aligned}
$$

where $\alpha+\beta=1(\alpha, \beta>0)$ and

$$
\begin{aligned}
m_{f 1}\left(x_{n}\right) & =A+B x_{n}+r, \quad x_{n} \leq t \\
& =A+B t+r, \quad x_{n}>t \\
m_{f 2}\left(x_{n}\right) & =A+B x_{n}+r .
\end{aligned}
$$

This has the effect of broadening the spectrum if $x_{n}$ exceeds some threshold, $t$. Such bimodal behaviour will be reflected in estimates of the mean Doppler shift and variance of the total spectrum, as given by (1). The measured values of $m_{f}\left(x_{n}\right)$ and $\sigma_{f}^{2}(x)$ can be written in terms of the individual means and variances of the two components of the bimodal spectrum, denoted $m_{f 1,2}(x)$ and $\sigma_{f 1,2}^{2}(x)$. In the absence of bimodal behaviour, $t \rightarrow \infty$ and the spectrum reverts to a single Gaussian shape.

The mean and variance of $\mathbf{G}\left(f, x_{n}, s_{1}, s_{2}\right)$ can be derived to assess the effect of any bimodal behaviour on estimates of these parameters from real data:

$$
\begin{aligned}
m_{f}\left(x_{n}\right)= & \int_{-\infty}^{\infty} f G\left(f, x_{n}, s_{1}, s_{2}\right) d f \\
= & \alpha m_{f 1}\left(x_{n}\right)+\beta m_{f 2}\left(x_{n}\right) \\
\sigma_{f}^{2}\left(x_{n}\right)= & \int_{-\infty}^{\infty}\left(f-m_{f}\left(x_{n}\right)\right)^{2} G\left(f, x_{n}, s_{1}, s_{2}\right) d f \\
= & \alpha\left(s_{1}^{2}+\left(\beta m_{f 2}\left(x_{n}\right)+(\alpha-1) m_{f 1}\left(x_{n}\right)\right)^{2}\right) \\
& \quad+\beta\left(s_{2}^{2}+\left(\alpha m_{f 1}\left(x_{n}\right)+(\beta-1) m_{f 2}\left(x_{n}\right)\right)^{2}\right) \\
= & \alpha s_{1}^{2}+\beta s_{2}^{2} \\
& \quad+\alpha \beta\left(m_{f 1}\left(x_{n}\right)^{2}+m_{f 2}\left(x_{n}\right)^{2}-2 m_{f 1}\left(x_{n}\right) m_{f 2}\left(x_{n}\right)\right) .
\end{aligned}
$$

In Section 2.4, the standard deviation of the spectrum was estimated from data as $\sigma_{f}\left(x_{n}\right)$, but it was found in general that this parameter was independent of $x_{n}$. Here the standard deviation of the spectrum width is modelled as being a random variable, $s$, that is independent of $x_{n}$. In $[21]$ the PDF of $s, p_{s}(s)$, is modelled as having a normal distribution, 
with mean $m_{s}$ and variance $\sigma_{s}^{2}$. The values of $s$ must of course be restricted to $s>0$. In practice it is found that $m_{s} \gg \sigma_{s}$ and so modelling $s$ in this way does not cause any problems. Now

$$
p_{s}(s)=\frac{1}{\sqrt{2 \pi} \sigma_{s}} \exp \left(\frac{-\left(x_{\mathrm{n}}-m_{s}\right)^{2}}{2 \sigma_{s}^{2}}\right) ; s>0
$$

Alternatively, the spectrum width can be modelled as having a gamma distribution, which is also a good fit to the data. Now

$$
p_{s}(s)=\frac{1}{\Gamma(\mu) c^{\mu}} s^{\mu-1} \exp \left(-\frac{s}{c}\right)
$$

where the shape, $\mu=m_{s}^{2} / \sigma_{s}^{2}$ and scale, $c=\sigma_{s}^{2} / m_{s}$.

\subsection{Bimodal data examples}

The bi-modal spectral model described in (6)-(8) can be described by four distinct parameters. These are the relative intensities of the components, $\alpha$ and $\beta=1-\alpha$, the centroid y-intercept $A$, the initial slope $B$ and the threshold, $t$. To estimate these parameters, a joint least squares fit to the centroid and variance has been implemented using the model described in (8) with the random variable, $r=0$. In addition to the 4 parameters above, the standard deviation $s=s_{1}=s_{2}$ is also estimated to provide a balanced fit. This parameter is not used in the final model (6), where it is instead realised as a random variable with separate models for its mean and variance. This model fitting was applied to a number of example data blocks at $30^{\circ}$ grazing from the dual-pol data set as shown in Figs. 7 and 8 with the fitted model parameters given in Table 2. Also shown in this table is the standard deviation, $\sigma_{r}$ of the fluctuation around the mean Doppler, $r$ as shown in (7), the mean and standard deviation of the spectral fluctuation, the time domain $\mathrm{CNR}$, the $\mathrm{K}$ distribution shape, $v$ and the spatial de-correlation length of the intensity, R. This measure of correlation is determined at the point where the absolute correlation falls to 1/e and is discussed further in Part 2 of this paper [25]

From these results, it can be seen that the bi-modal trends are strongest in the $\mathrm{HH}$ and $\mathrm{HV}$ upwind polarisations, where there is a change of slope for values of $x_{n}>t$. This behaviour is also present to a lesser extent in some of the other examples, such as the $\mathrm{HH}$ downwind where the standard deviation has a distinct upwards curve. Also of interest is the slope parameter $B$ which has larger positive values when looking up-wind, with larger negative values looking down-wind. The smallest absolute values of $B$ are found looking cross-wind. 
Table 2 Bi-Modal Example Fit Parameters for Dual-pol data

\begin{tabular}{|c|c|c|c|c|c|c|c|c|c|c|c|}
\hline \multirow{2}{*}{ Direction } & \multirow{2}{*}{ Pol. } & \multicolumn{4}{|c|}{$m_{f}\left(x_{n}\right)$} & \multirow{2}{*}{$\sigma_{r}(\mathrm{~Hz})$} & \multirow{2}{*}{$m_{s}(\mathrm{~Hz})$} & \multirow{2}{*}{$\sigma_{s}(\mathrm{~Hz})$} & \multirow{2}{*}{ CNR (dB) } & \multirow{2}{*}{$v$} & \multirow{2}{*}{$\mathbf{R}(\mathbf{m})$} \\
\hline & & $\mathbf{A}(\mathrm{Hz})$ & B (Hz) & $\mathbf{t}$ & $\beta$ & & & & & & \\
\hline \multirow{3}{*}{ Up-wind } & $\mathrm{HH}$ & -59.54 & 58.06 & 2.21 & 0 & 30.47 & 55.48 & 19.60 & 9.92 & 2.23 & 3.54 \\
\hline & $\mathrm{HV}$ & -42.22 & 115.24 & 1.22 & 0 & 32.52 & 74.97 & 21.45 & 5.51 & 5.11 & 3.16 \\
\hline & VV & 0.95 & 2.90 & - & 1 & 21.10 & 51.69 & 15.90 & 22.05 & 40.09 & 4.02 \\
\hline \multirow{3}{*}{ Cross-wind } & $\mathrm{HH}$ & -10.02 & 10.20 & 3.19 & 0.93 & 39.86 & 48.66 & 19.13 & 6.90 & 1.88 & 2.21 \\
\hline & $\mathrm{HV}$ & 49.14 & -43.88 & 1.26 & 0.21 & 38.39 & 46.34 & 18.24 & 3.80 & 4.70 & 2.44 \\
\hline & VV & -7.56 & 5.09 & - & 1 & 17.45 & 48.55 & 10.70 & 15.07 & 19.09 & 2.89 \\
\hline \multirow{3}{*}{ Down-wind } & $\mathrm{HH}$ & 21.13 & -34.52 & 1.89 & 0.54 & 27.70 & 49.98 & 20.35 & 7.88 & 4.75 & 2.43 \\
\hline & $\mathrm{HV}$ & 39.38 & -77.86 & - & 1 & 29.21 & 57.68 & 18.85 & 4.83 & 11.10 & 2.17 \\
\hline & VV & -1.31 & 4.17 & 0.96 & 0 & 21.25 & 47.06 & 13.05 & 21.22 & 27.62 & 3.76 \\
\hline
\end{tabular}

It can be seen that the spectrum width, $\sigma_{f}\left(x_{n}\right)$, and the mean Doppler shift, $m_{f}\left(x_{n}\right)$, both show considerable fluctuations. The variation in the mean Doppler frequency, $m_{f}\left(x_{n}\right)$, about the modelled local mean is obtained by subtracting the fitted trend in (6) from the data. As seen in the top part of Fig. 9, the distribution is approximately normal with a standard deviation of $30.47 \mathrm{~Hz}$.

The fluctuation in the spectrum width, $\sigma_{f}\left(x_{n}\right)$, is shown in the bottom part of Fig. 9 with a gamma distribution fit overlaid in red. This fluctuation can also be well modelled by a Gaussian distribution, subject to the restriction that $\sigma_{f}\left(x_{n}\right)>0$. In either case, the fit of the data can be characterised by estimating the mean, $m_{s}$, and standard deviation, $\sigma_{s}$ which are $55.48 \mathrm{~Hz}$ and $19.60 \mathrm{~Hz}$ for this example. For the gamma distribution, this corresponds to a shape, $\mu=8.01$ and scale, $c=6.92$.
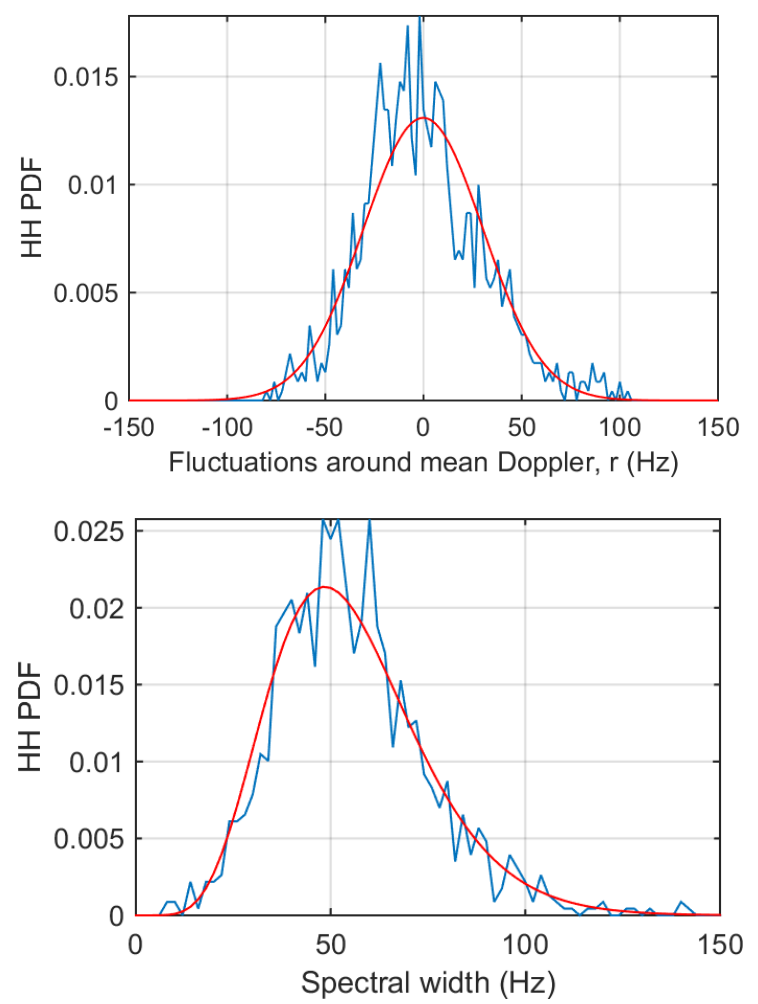
Figure 9 HH upwind PDFs for the mean Doppler (top) and spectral width (bottom). Blue - data, red - model fit.

\section{Conclusions}

This paper has shown that the Doppler spectra of radar sea clutter data collected at multiple polarisations, multiple look directions and at medium grazing angles can be represented by the modelling method originally reported in $[21]$ The analysis of the Ingara data sets described in this paper shows that the Doppler spectra of sea clutter can be expected to be highly variable, both spatially and temporally, for a wide range of environmental conditions and radar polarisations. This variability can be described in terms of two main characteristics. Firstly, the width of the power spectra, expressed as the standard deviation of the Doppler frequencies, fluctuates over range and time in a manner that is mainly independent of the local spectrum intensity. Secondly, the mean Doppler shifts of the local spectra, show a distinct correlation with local spectrum intensity. This is particularly evident when looking up or down wind, but is also observed to varying degrees for most look directions.

As a refinement of the model, it was observed that in some conditions a further broadening of spectra was observed when the local clutter intensity exceeded some threshold level, resulting in apparently bimodal spectra. This bimodal behaviour has also been captured in the model and the paper describes this behaviour can be modelled.

Part 2 of this paper [25] shows how the essential characteristics of the Doppler spectra, including their correlations over range and time, can be represented realistically in the model. It is further shown that these models can be used in analytic modelling of radar performance and in the simulation of realistic coherent clutter returns.

To be of value to a radar designer, models of clutter must include any dependencies on radar parameters and environmental conditions. The methodology for capturing this variation has been described in [31] and has been extended in Part 2 to also model the bimodal parameters.

\section{Acknowledgement}

This work was undertaken as part of the NATO SET-185 Task Group. We also want acknowledge the DSTO for providing the Ingara data sets.

\section{References}

[1] Ward, K.D., and Watts, S., Use of sea clutter models in radar design and development, IET Radar Sonar Navig.,Vol. 4, Iss. 2, April 2010, pp. 146 - 157 (Special Issue on Radar Clutter)

[2] Skolnik, M.I.,, Radar Handbook, Third Edition, McGraw Hill, 2008

[3] Nathanson, F.E., Radar Design Principles, McGraw Hill, 1969

[4] Long, M.W., Radar reflectivity of land and sea, Artech House, 1983 
[5] Ward, K. D., Tough, R. J. A., and Watts, S., "Sea Clutter: Scattering, the K Distribution and Radar Performance”, Institution of Engineering and Technology, 2nd Edition, 2013

[6] Sangston, K.J., Gini, F., Greco, M.S., Coherent Radar Target Detection in Heavy-Tailed Compound-Gaussian Clutter, IEEE Trans AES, Vol. 48, Issue 1, Jan 2012, 64 - 77.

[7] Rosenberg, L., and Bocquet, S., 'The Pareto Distribution for High Grazing Angle Sea-Clutter', IEEE Geoscience and Remote Sensing Symposium, 2013., pp 4209-4212

[8] Rosenberg, L. \& Bocquet, S., Application of the Pareto plus noise distribution to medium grazing angle seaclutter, IEEE Journal of Selected Topics in Applied Earth Observations and Remote Sensing, 2015, 8(1), 255261

[9] Rosenberg, L., Watts, S., Bocquet, S., 'Application of the K+Rayleigh Distribution to High Grazing Angle SeaClutter', International Conference Radar 2014, October 2014.

[10] Wetzel, L.B., Sea clutter, Chapter 15 of "Radar Handbook", M.I. Skolnok, Ed., Third Edition, McGraw Hill, 2008 .

[11] Davidson, G., Simulation of coherent sea clutter, IET Radar Sonar Navig.,Vol. 4, Iss. 2, April 2010, pp. 168 177 (Special Issue on Radar Clutter)

[12] Miller, R. J., Variability in spectra of low-grazing angle sea clutter returns, NATO/RTO Publications, Proceedings of SET Symposium on Low Grazing Angle Clutter: Its Characterisation, Measurement and Application, April 2000

[13] Chae, C.-S., and Johnson, J.T., A study of sea surface range-resolved Doppler spectra using numerically simulated low-grazing-angle backscatter data, IEEE Trans.on Geoscience and Remote Sensing Symposium, Vol. 52, No. 6, June 2013, pp 3452-3460

[14] Walker, D., Experimentally motivated model for low grazing angle radar Doppler spectra of the sea surface, IEE Proc. Radar, Sonar \& Navig., Vol. 147, No. 3, June 2000, pp 114-120.

[15] Walker, D., Doppler modelling of radar sea clutter, IEE Proc. Radar, Sonar \& Navig., Vol. 148, No. 2, April 2001, pp 73-80.

[16] Ward, K. D., Baker, C. J. and Watts S., Maritime surveillance radar part 1: Radar scattering from the ocean surface, Proc IEE, Vol 137, part F, No.2, April 1990 pp 51-62.

[17] Ritchie, M. A.,. Woodbridge, K. and Stove, A. G., Analysis of Sea Clutter Distribution Variation with Doppler using the Compound K-Distribution, IEEE Radar 2010 Int. Conf., May 2010, pp 495 - 499. 
[18] Greco, M., Stinco, P., Gini, F., and Rangaswamy, M., Impact of sea clutter nonstationarity on disturbance covariance matrix estimation and CFAR detector performance, IEEE Trans AES, Vol. 47, Issue 3, July 2010, pp $1502-1513$.

[19] Greco, M., Bordoni, F., Gini, F., "X-band Sea Clutter Non-Stationarity: The influence of Long Waves," IEEE Journal on Ocean Engineering, Special Issue on "Non-Rayleigh Reverberation and Clutter", Vol.29, No. 2, April 2004, pp.269-283

[20] Watts, S., "A new method for the simulation of coherent sea clutter," IEEE Radar Conference, pp. 52-57, May 2011

[21] Watts, S., Modeling and simulation of coherent sea clutter, IEEE Trans AES Vol. 48, No.4, October 2012, pp. $3303-3317$

[22] Watts, S., The effects of covariance matrix mismatch on adaptive CFAR performance, International Conference Radar 2013, Adelaide, September 2013

[23] Ritchie, M. A.. Stove, A. G., Watts, S., Woodbridge, K., and. Griffiths, H. D., Application of a New Sea Clutter Doppler Model, International Conference Radar 2013, Adelaide, September 2013.

[24] Watts, S., Rosenberg, L., and Ritchie, M., "Characterising the Doppler spectra of high grazing angle sea clutter,” in IEEE International Radar Conference, 2014.

[25] Watts, S., Rosenberg, L., Ritchie, M. and Bocquet, S., The Doppler Spectra of Medium Grazing Angle Sea Clutter; Part2: Model Assessment, IET RSN (part 2 of this paper)

[26] Crisp, D.J., Stacy, N.J., and Goh, A.S., "Ingara medium-high incidence angle polarimetric sea clutter measurements and analysis,” DSTO, Technical Report DSTO-TR-1818, February 2006.

[27] Quegan, S., "A unified algorithm for phase and cross-talk calibration of polarimetric data: Theory and observations,” IEEE Transactions on Geoscience and Remote Sensing, vol. 32, no. 1, pp. 89-99, 1994.

[28] Rosenberg, L., Crisp, D.J., Stacy, N.J., 'Analysis of the KK-distribution with medium grazing angle seaclutter', IET Radar Sonar and Navigation, Vol. 4, No. 2, pp 209-222., 2010.

[29] Bocquet, S., Rosenberg, L., Watts, S., 'Simulation of coherent sea clutter with inverse gamma texture', International Conference Radar 2014, October 2014.

[30] Rosenberg, L., "Characterisation of high grazing angle X-band sea-clutter Doppler spectra”, IEEE Trans. AES, January 2014 .

[31] Rosenberg, L., Watts, S., Bocquet, S. and Ritchie, M. "Data Characterisation of the Ingara HGA Dataset”, IEEE International Radar Conference, 2015. 